\title{
Philosophiques
}

\section{Violence de masse et sécession comme réparation : le cas du Kosovo}

\section{Philippe Roseberry}

Volume 39, numéro 2, automne 2012

Territorialité, identité nationale et justice mondiale

URI : https://id.erudit.org/iderudit/1013695ar

DOI : https://doi.org/10.7202/1013695ar

Aller au sommaire du numéro

\section{Éditeur(s)}

Société de philosophie du Québec

ISSN

0316-2923 (imprimé)

1492-1391 (numérique)

Découvrir la revue

Citer cet article

Roseberry, P. (2012). Violence de masse et sécession comme réparation : le cas du Kosovo. Philosophiques, 39(2), 421-434. https://doi.org/10.7202/1013695ar
Résumé de l'article

L'interprétation d'un acte de violence de masse est toujours délicate puisqu'elle confère un certain statut au groupe visé. Ce statut peut devenir un facteur important dans la décision de la communauté internationale de reconnaître ou non l'indépendance d'un groupe et de son territoire. Cet article examine le cas de la reconnaissance du Kosovo par la communauté internationale, en février 2008, et soutient que cette reconnaissance a été rendue possible par

l'utilisation d'arguments basés sur le statut collectif de victime de nettoyage ethnique détenu par la population albanaise du Kosovo. L’article comble le fossé entre, d'un côté, la théorie de la sécession comme réparation, et de l'autre, les travaux sur la violence de masse contre les groupes ethnonationaux. L'analyse montre que la pratique de la reconnaissance étatique a été réorientée vers un critère restrictif lié au type de violence subie par le groupe réclamant l'indépendance. Dans cette optique, le territoire devient une forme tangible de compensation pour les injustices subies, ce qui tranche par rapport à la pratique antérieure de la reconnaissance étatique dans l'ex-Yougoslavie et ailleurs.
Ce document est protégé par la loi sur le droit d'auteur. L'utilisation des services d’Érudit (y compris la reproduction) est assujettie à sa politique d'utilisation que vous pouvez consulter en ligne.

https://apropos.erudit.org/fr/usagers/politique-dutilisation/ 


\title{
Violence de masse et sécession comme réparation: le cas du Kosovo'
}

\author{
PHILIPPE ROSEBERRY \\ Département d'études politiques, Queen's University, Kingston, Canada \\ philippe.roseberry@queensu.ca
}

\begin{abstract}
RÉSUMÉ. - L'interprétation d'un acte de violence de masse est toujours délicate puisqu'elle confère un certain statut au groupe visé. Ce statut peut devenir un facteur important dans la décision de la communauté internationale de reconnaître ou non l'indépendance d'un groupe et de son territoire. Cet article examine le cas de la reconnaissance du Kosovo par la communauté internationale, en février 2008 , et soutient que cette reconnaissance a été rendue possible par l'utilisation d'arguments basés sur le statut collectif de victime de nettoyage ethnique détenu par la population albanaise du Kosovo. L'article comble le fossé entre, d'un côté, la théorie de la sécession comme réparation, et de l'autre, les travaux sur la violence de masse contre les groupes ethnonationaux. L'analyse montre que la pratique de la reconnaissance étatique a été réorientée vers un critère restrictif lié au type de violence subie par le groupe réclamant l'indépendance. Dans cette optique, le territoire devient une forme tangible de compensation pour les injustices subies, ce qui tranche par rapport à la pratique antérieure de la reconnaissance étatique dans l'ex-Yougoslavie et ailleurs.
\end{abstract}

\begin{abstract}
The interpretation of an act of mass violence is always a contentious issue since it confers a certain status to the targeted group. This status may become an important factor in the international community's decision to recognize or not the independence of a group and its territory. This article examines the case of Kosovo's February 2008 recognition by the international community and argues that this recognition was made possible by the summoning of arguments based on Kosovo Albanians' collective status as victims of ethnic cleansing. The article bridges the gap between on the one hand the Remedial Right theory of secession, and on the other hand, works on mass violence against ethnonational groups. The article shows that the practice of state recognition was reoriented towards a restrictive criterion linked to the type of violence sustained by the group claiming independence. Under this interpretation, territory becomes a tangible form of compensation for suffered injustices, which contrasts with earlier practice of state recognition in the former Yugoslavia and elsewhere.
\end{abstract}

Catégoriser un acte de violence de masse est toujours une opération contentieuse. Cela l'est d'autant plus lorsque cette catégorisation s'opère en tandem avec un débat sur la reconnaissance étatique d'un groupe sécessionniste ter-

1. Une première version de cet article a été présentée au colloque "Territorialité, identité nationale et justice mondiale", organisé par le Centre de recherche interdisciplinaire sur la diversité au Québec (CRIDAQ). Je remercie Michel Seymour, Christine Straehle, FrédérickGuillaume Dufour et tous les autres participants pour leurs commentaires. 
ritorialement concentré. Pour l'analyse légale, qualifier un acte de génocide, plutôt que de nettoyage ethnique ou de crime de guerre, dépend essentiellement de définitions juridiques dont l'objectif est de démontrer la responsabilité criminelle d'un État. Mais comme le montre cette analyse, la façon dont une campagne de violence de masse est perçue et catégorisée par différents acteurs politiques affecte aussi les perspectives du groupe visé par la violence de se voir reconnaître comme État indépendant. Cette reconnaissance est alors présentée comme une réparation pour les injustices subies. Le cas de la déclaration unilatérale d'indépendance du Kosovo, en 2008, fournit d'importantes clés de compréhension de ce phénomène.

Interpréter et catégoriser la violence perpétrée par l'État permet de clarifier le statut du groupe visé par la violence. Ce statut varie considérablement selon que l'on utilise la catégorie de victime de "nettoyage ethnique ", plutôt que celle, négativement connotée, "d'insurgé ", pour décrire le groupe visé. Cette distinction n'est pas que sémantique, car elle souligne la performativité inhérente du langage associé à l'identification et à la catégorisation des actes de violence de masse. Cette catégorisation nous en dit long sur le type de réclamation dont les victimes peuvent se prévaloir, ainsi que sur le soutien qu'elles peuvent espérer recevoir de la communauté internationale.

Le cas de la reconnaissance internationale du Kosovo en tant qu'État indépendant représente un jalon important du débat théorique et politique concernant les conditions dans lesquelles l'indépendance est concédée à un État, car il lie les débats sur la catégorisation de la violence de masse à ceux concernant les bases légitimes de la sécession et de la reconnaissance étatique. Le statut final du Kosovo constituait, au moins jusqu'à sa déclaration unilatérale d'indépendance, le I7 février 2008, une question épineuse pour les organisations internationales et les États ayant soutenu l'autonomie de facto de l'ex-province serbe à la suite du conflit de r 998-I999. La communauté internationale, c'est-à-dire les membres du Conseil de sécurité de l'ONU - ainsi que les principaux membres de l'OTAN, qui participèrent à la campagne aérienne contre la Serbie en I999 et reconnurent l'indépendance du Kosovo en 2008 , faisait face à un double dilemme ${ }^{2}$. D'un côté, il semblait impossible d'envisager un retour de l'appareil sécuritaire et administratif serbe au Kosovo. De l'autre, il apparaissait aussi très délicat d'ouvrir la porte à la reconnaissance d'une déclaration unilatérale d'indépendance étant donné l'extrême sensibilité de la question du droit des minorités nationales dans l'Europe de l'Est contemporaine. Dans ce contexte, la question se posait de justifier l'indépendance du Kosovo de la manière la plus convaincante, mais aussi la moins déstabilisante.

Cette analyse identifie le concept de sécession "comme réparation", basé sur la violence de masse subie par la population albanaise du Kosovo,

2. Au moment de la publication, quatre-vingt-onze pays avaient reconnu l'indépendance du Kosovo. 
comme une justification particulièrement efficace par laquelle les puissances administratrices engagées dans la gestion du protectorat kosovar ont cherché à convaincre, et se convaincre, de la légitimité de l'indépendance du Kosovo. À travers une lecture particulière du type de violence subie par la population albanaise du Kosovo, la communauté internationale a établi un lien sans précédent entre type de violence de masse et droit à la sécession. Cela amène à voir la sécession comme remède à des atrocités subies et modifie le rôle du territoire dans ce processus, celui-ci passant de base de l'État démocratique à un bien échangeable en vue d'une réparation. Bien que ce lien ait fourni aux puissances administratrices les outils conceptuels pour régler la question du statut final du Kosovo, il a aussi élevé le seuil au-delà duquel les demandes sécessionnistes en situation de conflit violent sont recevables. Notre analyse recense d'abord la littérature contemporaine sur la catégorisation de la violence de masse et identifie les implications de celles-ci pour l'éthique de la sécession. Tirant profit de ces indications théoriques, elle replace ensuite le lien entre violence de masse et sécession, ainsi que le cas du Kosovo, dans une perspective comparée. Elle examine le discours de certains acteurs internationaux engagés dans la légitimation du statut final du Kosovo en tant qu'État souverain dans le but de comprendre la logique de la reconnaissance. L'analyse conclut avec une discussion des implications théoriques et pratiques du "précédent » du Kosovo pour l'enjeu global de la sécession.

\section{Violence de masse et sécession comme réparation: réflexions théoriques}

\section{Interpréter la violence de masse}

Le lien que nous avons établi entre violence subie et statut final du Kosovo prend racine dans un courant de recherche émergent dont l'objectif est l'adoption de catégories analytiques sociologiques, plutôt que légales, dans l'étude de la violence de masse. Jacques Sémelin et Benjamin Valentino présentent de tels efforts de catégorisation basés sur la logique stratégique de l'appareil sécuritaire et militaire de l'État perpétrateur ${ }^{3}$. Les auteurs développent une typologie de la logique de la violence de masse qui comprend deux catégories principales basées sur le résultat escompté de la violence.

Par "détruire pour soumettre», Sémelin désigne une catégorie d'actes de violence de masse dont l'objectif est la sujétion, temporaire ou permanente, du groupe visét. S'il y a de toute évidence destruction intentionnelle d'une fraction du groupe visé, cette destruction est perçue comme nécessaire à l'imposition ou au rétablissement de l'ordre par l'État. Le plus souvent, dans cette situation, l'État cherche à briser une insurrection, réelle ou imaginée, comme les armées révolutionnaires françaises l'ont fait en Vendée, dès

3. Sémelin, 2005; Valentino, 2004.

4. Sémelin, 2005, p. 389-390. 
I793, ou plus récemment en Algérie dans le cadre de la lutte contre le Front de libération nationale ${ }^{5}$. Valentino s'exprime en termes similaires en identifiant une catégorie générale de la violence de masse "coercitive» qui inclut les luttes anti-insurrectionnelles ${ }^{6}$.

Cette première catégorie se distingue à la fois chez Sémelin et chez Valentino d'une seconde logique désignée par la dénomination "détruire pour éradiquer». Là où la catégorie précédente désigne des actes aux fins plus limitées, la seconde renvoie à une campagne visant « l'élimination d'une collectivité d'un territoire plus ou moins vaste, contrôlé ou convoité par un État $^{7}{ }$. Sémelin distingue de plus, au sein de cette même catégorie, l'action génocidaire, visant la destruction physique de l'ensemble du groupe visé, du nettoyage ethnique, par lequel une violence ciblée est utilisée afin d'encourager la fuite du reste du groupe vers l'extérieur du territoire convoité ${ }^{8}$. Valentino définit une catégorie similaire en présentant la catégorie des massacres "dépossessifs » et distingue lui aussi entre nettoyage ethnique et éradication totale.

Ces distinctions théoriques liées à l'instrumentalité de la violence sont utiles au débat scientifique sur le statut final des territoires contestés. Si la recherche ne doit pas contourner l'enjeu évident de la responsabilité morale et légale de l'État dans les cas de violence de masse, elle doit aussi être consciente que les groupes territorialement concentrés visés par une violence significative de l'État se trouvent dans une situation bien différente lors des négociations avec l'État, le groupe majoritaire et les acteurs internationaux concernés. Le statut détenu par un groupe visé contient une dimension symbolique et stratégique. La violence, récente ou ancienne, devient un «enjeu de mémoire» qui peut être mobilisé lors de négociations sur la propriété d'un territoire donnés.

Les parties soumises directement ou indirectement aux actes de violence de masse entretiennent donc des attentes concrètes quant aux implications du statut de victime. Une hiérarchie des crimes et de la souffrance s'établit dans laquelle "détruire pour éradiquer» prend le pas sur «détruire pour soumettre ". Un groupe s'insurgeant contre la domination réelle ou perçue d'un groupe majoritaire ou d'un État ne peut s'attendre à bénéficier des mêmes réparations que celui visé par une campagne non provoquée de nettoyage ethnique, voire de génocide.

Dans le cas du Kosovo, le débat sur la nature du conflit de I998-I999 entre insurgés albanais et forces de sécurité serbes continue de diviser les analystes. Si certains y voient un cas évident de nettoyage ethnique contre la

\footnotetext{
5. Ibid., p. 391-392.

6. Valentino, 2004, p. 70; I96-I97.

7. Sémelin, 2005 , p. 398.

8. Ibid., p. 405 .

9. Sémelin, 2005 , p. 3 Io.
} 
population albanaise ${ }^{10}$, voire même de génocide ${ }^{11}$, d'autres interprètent le conflit comme une lutte anti-insurrectionnelle certes disproportionnellement violente, mais partiellement provoquée par l'Armée de libération du Kosovo $^{12}$. Il ne s'agit pas ici de prendre position en faveur d'une interprétation particulière, mais de démontrer qu'en choisir une plutôt que l'autre a des implications différentes pour le contrôle d'un territoire convoité par un État et un groupe sécessionniste.

\section{Injustices subies et sécession comme réparation}

Ayant établi que le statut dont dispose un groupe victime de violence de masse influe sur sa capacité de recevoir le soutien de tierces parties, il s'agit de lier ce statut aux travaux portant sur l'éthique de la sécession. Ceux-ci nous éclairent quant au fait que les demandes politiques formulées lors de débats sur la reconnaissance étatique sont particulièrement fortes lorsqu'elles se basent sur le statut de victime de violence de masse. Ces demandes se réfèrent souvent à l'argument de la sécession comme réparation.

Suivant la pensée d'Allen Buchanan, on peut ordonner le champ des théories normatives de la sécession en deux grandes familles ${ }^{13}$, soit les théories du droit primaire, et celle du droit à la réparation ${ }^{14}$. Les théories du droit primaire conçoivent le droit à la sécession comme découlant de caractéristiques objectives partagées par une population ou un groupe, comme la langue, la religion ou la nationalité ${ }^{15}$. Dans une autre version de la théorie, le droit à la sécession découle plutôt de l'existence d'associations volontaires d'individus souhaitant l'indépendance, dont le désir est soumis à un référendum, parfois selon des règles constitutionnelles préétablies ${ }^{16}$.

À l'inverse, la théorie de la sécession comme réparation repose sur le principe général selon lequel un groupe ne peut faire sécession que lorsqu'il a subi certaines injustices graves pour lesquelles la sécession est le remède approprié de dernier recours ${ }^{17}$. Buchanan inclut justement parmi ces injustices la menace ou l'utilisation effective de moyens mettant en danger la survie du groupe visé, comme le nettoyage ethnique ou le génocide. Dans des travaux plus récents, Buchanan ajoute que l'annexion illégitime d'un territoire, comme l'incorporation forcée des États baltes à l'Union sovié-

10. Judah, 2002.

11. Power, 2002

12. Brubaker, 2004, p. I3-I 5 .

13. Cette analyse ne doit pas être interprétée comme mettant de l'avant une théorie normative de la sécession. Elle cherche plutôt à comprendre comment les arguments contenus dans ces théories informent les décisions des acteurs de la reconnaissance étatique.

14. Buchanan, I997, I998.

15. Ibid., p. 38

16. Ibid., p. 38-39; Beran, I980. Voir Weinstock, 200I, sur l'inclusion d'une formule de sécession dans la constitution d'un État.

17. Buchanan, I997, p. 34-35 
tique, ainsi que la violation récurrente de l'autonomie territoriale d'un groupe au sein d'un État englobant constituent aussi des griefs suffisants pour justifier la sécession comme réparation ${ }^{18}$. Dans cette théorie de la sécession, le rôle du territoire change de façon importante puisqu'il passe d'une base physique sur laquelle peut s'ériger un nouvel État démocratique à celui de bien compensatoire censé remédier à une injustice commise par l'État englobant. Bien qu'il ne soit pas exclu que le nouvel État soit gouverné de façon démocratique, ce critère n'est plus une condition nécessaire autorisant la sécession. Il est frappant de noter la similarité de ce discours avec celui de la communauté internationale au sujet du Kosovo, en ce sens que les puissances administratrices ont insisté à la fois sur le statut particulier des Albanais du Kosovo et sur le caractère de dernier recours de l'indépendance du Kosovo pour justifier leur décision ${ }^{19}$.

La théorie de la sécession comme réparation comporte toutefois un certain nombre de limitations en tant que composante d'un cadre analytique. D'abord, celle-ci est fondamentalement imprécise en ce qui a trait au type et à l'étendue des injustices qui justifient la sécession. Si Buchanan mentionne explicitement les actions menaçant la survie physique d'un groupe, ce qui sous-entend la présence de violence, il semble plus difficile d'établir des paramètres clairs qui séparent l'injustice significative de celle qui l'est moins ${ }^{20}$.

De plus, les théories normatives de la sécession, au sens général, ne spécifient pas quelle justification de la sécession sera mise de l'avant par les parties concernées lorsque plusieurs sont disponibles. Au Kosovo, la quasitotalité des justifications potentielles de la sécession, autant primaires que "réparatrices", étaient revendicables. En I990 et I99I, l'Assemblée du Kosovo ainsi que la population dans son ensemble exprimèrent leur désir quasi-unanime d'indépendance complète du Kosovo, satisfaisant ainsi le critère de base des théories primaires de la sécession ${ }^{21}$. Par ailleurs, la recherche cite la suppression du statut de province autonome du Kosovo par le pouvoir central en 1987, ainsi que l'imposition de la loi martiale dans les années I980 et I990 comme preuve du non-respect de l'autonomie interne du Kosovo, un grief justifiant une autre version de la théorie de la sécession comme réparation. Pourtant, les Nations Unies et les principales puissances impliquées dans l'administration du Kosovo optèrent pour une troisième option et choisirent d'interpréter la sécession comme réparation sur la base de catégories issues du langage de la violence de masse. Cette décision relève d'un choix stratégique et non d'un geste aléatoire.

18. Buchanan, 2003a, p. 218; 2003 b, p. 247-248.

19. Envoyé spécial du secrétaire général des Nations-Unies, 2007, p. 2.

20. Buchanan, I997, p. 37

21. Janjić, Lalaj et Pula, 2009. 
Bien qu'il soit très difficile de déterminer objectivement le niveau de violence au-delà duquel la sécession devient une mesure de réparation justifiée, notamment parce qu'il est mesuré d'après les perceptions intersubjectives des acteurs engagés dans le débat, il semble clair que, dans le cas du Kosovo, la communauté internationale a élevé la barre à un niveau supérieur. On peut en fait imaginer un continuum d'injustices allant de la répression des institutions culturelles à la violence sporadique et ciblée, jusqu'à la violence massive et récurrente. Plus l'on s'approche de l'échelon supérieur, plus la sécession devient normativement acceptable pour la communauté internationale. À un niveau inférieur de l'échelle, l'État cherche à reprendre le contrôle d'une population par une violence exemplaire. La communauté internationale sanctionne l'usage disproportionné de la violence par l'État, mais respecte son intégrité territoriale puisque l'enjeu demeure la sécurité intérieure. Plus haut dans l'échelle, en revanche, l'objectif de l'État est de dépeupler un territoire en vue d'en prendre le contrôle. La sécession du territoire visé devient alors une option à considérer puisque la population ciblée par la violence ne peut être réintégrée dans l'État et doit recevoir compensation pour les atrocités subies.

Pour la communauté internationale, catégoriser la violence au Kosovo comme un cas de contre-insurrection disproportionnellement violente n'aurait pas permis de justifier l'indépendance de celui-ci sans courir le risque d'encourager des mouvements insurrectionnels sécessionnistes similaires dans la région. L'action des forces de sécurité serbes au Kosovo a dû être re-catégorisée en tant que nettoyage ethnique, un terme plus normativement chargé qui a ouvert la porte à la sécession comme réparation.

\section{Ressources mémorielles et demandes politiques en perspective comparée}

Le fait que la mémoire de la violence subie soit utilisée comme ressource stratégique lors de négociations sur la reconnaissance étatique ne devrait pas être surprenant. Un regard comparatif sur l'usage stratégique de la mémoire de la violence de masse révèle la relative fréquence d'une telle stratégie dans des contextes variés. Lors de la décolonisation, les élites révolutionnaires soulignèrent avec force le lien entre règne colonial et violence de masse afin de soutenir leurs revendications contre les puissances européennes. Mais comme on le remarque, même si l'idée de réparation apparaît dans les débats sur la décolonisation, elle est rarement liée à un droit à la sécession ${ }^{22}$. Puisque les colonies des puissances européennes ne faisaient que rarement partie intégrante du territoire métropolitain, sauf dans le cas de l'Algérie française, celles-ci n'ont, à proprement parler, pas fait sécession de leur empire colonial respectif. 
Plus récemment, dans le Rwanda post-génocide, le régime du Président Paul Kagame semble bénéficier de la complaisance internationale sur la question des droits humains et politiques étant donné son statut de défenseur des victimes du génocide de $1994^{23}$. Sur un autre continent, en Ukraine, certains des chefs de file de la «révolution orange» se sont approprié la mémoire de la famine de I932-I933 (Holodomor) et l'ont utilisée dans leur lutte contre des adversaires domestiques jugés pro-russes, ainsi que dans leurs relations tendues avec la Russie ${ }^{24}$. En Bosnie-Herzégovine, des élites bosniaques ont utilisé la mémoire des massacres de I 992 à I 995 afin de soutenir l'idée que tout renforcement de l'autonomie de l'unité territoriale serbe de Bosnie équivaudrait à approuver tacitement le génocide perpétré contre certains segments de la population musulmane pendant la guerre ${ }^{25}$.

On doit noter, toutefois, que l'usage stratégique de la mémoire de la violence de masse est généralement l'instrument d'élites étatiques engagées dans la consolidation d'un État déjà indépendant, ce que Rogers Brubaker a appelé un «État nationalisant ${ }^{26}$ ». Dans le cas bosniaque, par exemple, l'invocation de la mémoire des atrocités du temps de guerre constitue une manœuvre visant à affaiblir des adversaires domestiques dans le contexte de réformes constitutionnelles. Le cas du Kosovo, par contraste, reflète une tentative de la communauté internationale de justifier l'indépendance d'un territoire non souverain dont le statut est contesté sur le plan domestique et international.

La même dynamique se reproduit lors de crises internationales majeures, telles que l'intervention des États-Unis et des pays de l'Union européenne dans les guerres de succession de l'ex-Yougoslavie, où l'on observe le rôle important joué par la mémoire de la violence de masse dans l'attitude des puissances étrangères quant au statut des populations visées par la violence. David Campbell et V. P. Gagnon soutiennent que la catégorisation essentialiste de la guerre en Bosnie-Herzégovine comme conflit ethnique insoluble explique largement la nature timide et limitée de l'intervention internationale dans le conflit entre avril 1992 et l'été $1995^{27}$. Il a fallu attendre le bombardement du marché de Sarajevo, en I994, ainsi que les massacres de Goražde et Srebrenica, en juillet I995, dans les zones de sécurité de l'ONU pour que la communauté internationale concède au groupe musulman le statut de principale victime du conflit. Cette décision permit ensuite l'utilisa-

23. Lemarchand, 2002; Reyntjens, 2006.

24. Arel, 2006.

25. Catić, 2008.

26. Brubaker, I996. Tous les cas majeurs d'utilisation stratégique de la mémoire de la violence de masse dans la période contemporaine concernent des États déjà indépendants. La liste inclut le Rwanda post-I994, l’Ukraine postsoviétique, l'Arménie postsoviétique et la BosnieHerzégovine.

27. Campbell, I997; Gagnon, 2004. 
tion de bombardements aériens contre les forces serbes de Bosnie ${ }^{28}$. Toutefois, dans ce cas, le problème pour la communauté internationale est de répondre aux violations systématiques des droits humains des populations civiles, et non de lier cet enjeu à un quelconque arrangement territorial. Au Kosovo, par contraste, les puissances administratrices ont débattu le statut territorial final du Kosovo en le liant explicitement à la question de la sécession comme réparation d'une injustice subie.

\section{Le discours de la communauté internationale sur la reconnaissance du Kosovo}

La spécificité du cas du Kosovo en termes de reconnaissance internationale issue d'une lecture particulière de la violence perpétrée contre la population albanaise du Kosovo est clairement identifiable dans les déclarations de reconnaissance présentées par les principales puissances administratrices dans les jours suivant la déclaration d'indépendance du Kosovo. La déclaration américaine, la plus influente et la plus attendue des autorités kosovares, établit sans ambages les bases normatives de la décision de reconnaître le Kosovo:

«Nine years ago, the international community, led by NATO, acted to end brutal attacks on the Kosovar Albanian population. This timely international intervention ended the violence, leading to a United Nations Security Council decision to suspend Belgrade's governance and place Kosovo under interim UN administration. [...] In light of the conflicts of the r 990 s, independence is the only viable option to promote stability in the region. ${ }^{29}$ "

Le secrétaire d'État, souhaitant souligner la nature singulière du cas du Kosovo, ajoute que la nécessité de reconnaître le Kosovo s'explique largement par un historique de conflit ethnique et de crimes contre les civils:

«The unusual combination of factors found in the Kosovo situation - including the context of Yugoslaviass breakup, the history of ethnic cleansing and crimes against civilians in Kosovo, and the extended period of UN administration - are not found elsewhere and therefore make Kosovo a special case. Kosovo cannot be seen as a precedent for any other situation in the world today. ${ }^{30}$ »

Déjà, les grandes lignes du raisonnement des puissances occidentales sont bien marquées. Bien qu'une référence aux institutions démocratiques du peuple kosovar ait été faite, ainsi qu'un rappel de la durée de l'administration onusienne au Kosovo, la diplomatie américaine juge nécessaire de faire appel à l'argument de la sécession comme réparation sur la base d'une interprétation de la violence en tant que cas de «nettoyage ethnique».

28. Burg et Shoup, I999, p. 407-4I2; Sobel, I998.

29. United States Department of State, 2008.

30. United States Department of State, 2008. 
Le point de vue canadien, quelques mois plus tard, va essentiellement dans le même sens que la déclaration américaine, soulignant non seulement la volonté des autorités kosovares d'aller de l'avant en ce qui concerne l'indépendance, mais aussi le fondement normatif de cette décision, c'est-à-dire l'historique de conflit ethnique:

Le Kosovo est un cas unique, comme en témoignent sa récente histoire, marquée par la guerre et l'épuration ethnique, le rôle joué subséquemment par l'ONU et l'OTAN dans l'administration et la sécurité du territoire, ainsi que le rôle constant que des organisations internationales, par exemple l'Union européenne, exerceront pour l'aider dans sa transition vers la pleine indépendance ${ }^{31}$.

Le gouvernement canadien souligne aussi le caractère sui generis du cas du Kosovo, ainsi que les circonstances spéciales qui rendent ce cas difficilement comparable à d'autres. Si certains observateurs ont interprété cette déclaration comme une référence à peine voilée aux débats constitutionnels canadiens, l'impact plus large de la déclaration est d'invalider le cas du Kosovo comme base de comparaison pour les demandes d'indépendance passées et futures en ex-Yougoslavie, et plus largement en Europe de l'Est.

La déclaration française fait quant à elle appel à des arguments plus divers, tout en identifiant fermement la base normative de l'indépendance du Kosovo dans l'historique de conflits violents des guerres de succession de l'ex-Yougoslavie. Bernard Kouchner, ministre des Affaires étrangères et ancien administrateur de la Mission des Nations Unies au Kosovo (MINUK), fait référence à la «rébellion» des Albanais du Kosovo contre l'État serbe, mais aussi aux «crimes et à la souffrance» infligés par les forces de sécurité serbes $^{32}$. Kouchner reconnaît aussi le caractère ultime de la concession de l'indépendance à un groupe ayant subi une violence de masse: "C'est la première fois dans l'histoire du monde qu'un tel conflit, non seulement voit la paix arriver au bout de quelques années, mais la solution, cette indépendance, qui est une exception. C'est une exception qui ne doit pas servir d'exemple ${ }^{33}$.»

On doit aussi considérer l'avis consultatif de la Cour internationale de justice $(\mathrm{CIJ})$ du 22 juillet 20 Io à la lumière du lien établi par la communauté internationale entre la violence subie par les Albanais du Kosovo et l'indépendance de ce territoire. Bien qu'il soit un repère légal important, l'avis de la Cour selon lequel la déclaration unilatérale d'indépendance du Kosovo ne viole pas le droit international existant ne discute pas directement les bases normatives de la sécession du Kosovo en 2008 . Toutefois, son interprétation de la violence menant au statut de protectorat onusien soutient l'interprétation de l'indépendance de ce territoire comme réparation des préjudices

31. Ministère des Affaires étrangères et du Commerce international, 2008.

32. Ministère des Affaires étrangères et européennes, 2008.

33. Ibid., 2008. 
infligés par l'État serbe. De plus, la Cour déclare qu'elle n'a pas à établir de droit positif à l'indépendance pour le Kosovo, mais seulement à en nier l'illégalité, ce qui s'oppose aux théories du droit primaire à la sécession, qui elles, conditionnent la sécession à un tel droit ${ }^{34}$. La Cour affirme aussi que l'administration intérimaire et le cadre légal établi par la résolution I 244 du Conseil de sécurité constituent une exception justifiée par l'occurrence d'un conflit violent au Kosovo ${ }^{35}$.

Dans tous les cas présentés plus haut, la justification de l'indépendance du Kosovo est inextricablement liée au statut « réparateur» de son territoire et à l'injustice subie par le groupe majoritaire qui l'occupe. Cette ligne de justification diffère sensiblement des épisodes précédents de reconnaissance étatique des États successeurs de l'ex-Yougoslavie, au cours des vingt dernières années. En I99I, la Communauté européenne établit la Conférence internationale sur l'ex-Yougoslavie pour coordonner sa politique dans la région. La Conférence avait alors identifié un certain nombre de critères pour guider la reconnaissance des États successeurs auto-déclarés de l'exYougoslavie, soit le principe de la tenue d'un référendum sur l'indépendance, l'introduction de protections légales pour les minorités nationales, ainsi que le respect des frontières internes yougoslaves déjà existantes ${ }^{36}$. En d'autres termes, la Conférence suivait alors les principes classiques de l'autodétermination, ainsi qu'une certaine forme de reconnaissance conditionnelle basée sur le respect du droit des minorités et le fonctionnement démocratique du territoire nouvellement indépendant.

$\mathrm{Au} \mathrm{Kosovo,} \mathrm{par} \mathrm{contraste,} \mathrm{le} \mathrm{retour} \mathrm{aux} \mathrm{critères} \mathrm{légaux} \mathrm{et} \mathrm{procéduraux}$ de la Conférence internationale sur l'ex-Yougoslavie ne semble par avoir été sérieusement considéré comme justification principale de la reconnaissance étatique. Cette décision illustre un changement important dans le traitement de l'enjeu de la reconnaissance étatique par des membres influents de la communauté internationale. L'héritage de la Conférence semble avoir été relégué au second plan au profit d'arguments fondés sur les mesures réparatrices devant être prises envers une population victime de violence de masse. Le territoire est alors devenu le bien échangeable le plus important en vue d'effectuer la réparation. Ce changement de cap est d'autant plus intriguant que les arguments utilisés pour reconnaître les nouveaux États est-européens au début des années I990 étaient toujours disponibles en 2008 pour justifier l'indépendance du Kosovo.

34. Cour internationale de justice, 20I0, paragr. 55-56.

35. Cour internationale de justice, 20I0, paragr. 97-99.

36. European Community, I99 Ia ; I99 Ib ; I99 Ic; Arbitration Commission of the International Conference on Yugoslavia, I992; Caplan, 2005, 64-7I. 


\section{Condusion: LeKosovo et la pratique de la reconnaissance étatique}

La façon dont un épisode de violence de masse contre un groupe territorialement concentré est perçue et catégorisée par des tierces parties internationales affecte de façon importante les perspectives de reconnaissance étatique du groupe visé. Cette constatation devient plus claire à mesure que l'analyse de la violence de masse s'affranchit des catégories légales au profit d'une analyse des logiques stratégiques. Dès lors que la façon dont la communauté internationale perçoit et interprète la violence a été identifiée, il devient possible d'évaluer l'étendue du soutien extérieur dont le groupe sécessionniste peut bénéficier. Là où la violence a été sévère, notamment dans les cas de nettoyage ethnique ou de génocide, la communauté internationale peut vraisemblablement concéder l'indépendance au groupe sécessionniste, mais en utilisant l'argument du transfert de territoire comme réparation, puisque celui-ci minimise le risque d'avalanche de nouvelles déclarations unilatérales d'indépendance.

Si le fait de baser des demandes d'indépendance sur la mémoire de la violence subie est intuitif autant pour les groupes sécessionnistes eux-mêmes que pour leurs défenseurs internationaux, comme le montre la littérature sur la sécession comme réparation, notre analyse suggère que la connexion entre violence subie et demandes sécessionnistes n'a pas été explicitée jusqu'à maintenant lors des épisodes antérieurs de reconnaissance étatique, ni dans la recherche les analysant. Par contraste, la communauté internationale a reconnu l'indépendance du Kosovo en 2008 en faisant explicitement référence à la répression violente des Kosovars par les forces de sécurité serbes en 1998 et I999.

Significativement, la communauté internationale n'a pas justifié sa décision sur la base de la suppression de l'autonomie territoriale du Kosovo dans les années I980 et I990. Elle ne l'a pas justifié non plus par le principe d'auto-détermination, par celui du droit des minorités ou encore par celui du respect des frontières internes de l'ex-Yougoslavie, comme ce fut le cas pour la reconnaissance des autres ex-républiques yougoslaves. Au lieu de cela, elle a présenté sa décision au regard du type de violence dont la population albanaise du Kosovo a été la cible, donnant ainsi un sens pratique au concept de sécession comme réparation. Les choix effectués par la communauté internationale démontrent la force et la crédibilité des arguments utilisant la mémoire de la violence subie, en plus d'illustrer le changement des pratiques discursives des États et des organisations engagés dans la reconnaissance étatique.

Notre analyse soutient donc que le cas du Kosovo représente une étape importante dans l'évolution du débat scientifique et politique entourant la reconnaissance étatique. Un lien évident a été établi par la communauté internationale entre violence subie et droit à l'indépendance, et cet argument s'est imposé face à d'autres permettant eux aussi de justifier l'indépendance du Kosovo. Le cas du Kosovo est aussi une étape importante, au sens où la 
communauté internationale a cherché à limiter la sécession légitime aux rares épisodes au cours desquels un État fait un usage disproportionné de la violence de masse contre un groupe concentré territorialement. En d'autres termes, la communauté internationale a élevé le seuil minimal d'injustices à un niveau considérablement plus élevé. Si le cas du Kosovo est un quelconque précédent, il est caractérisé par la limitation du droit à la sécession plutôt que par un soutien général et absolu à la sécession.

\section{Bibliographie}

Affaires étrangères et Commerce international Canada. 2008. «Le Canada se joint à la reconnaissance internationale du Kosovo », I 8 mars 2008, n. 59, Ottawa, Gouvernement du Canada [http://www.international.gc.ca/media/aff/newscommuniques/2008/385954.aspx ? lang=fra], consulté le i 5 janvier 2010.

Arbitration Commission of the International Conference on Yugoslavia. "Opinion No. 4 on Recognition of the Socialist Republic of Bosnia-Herzegovina by the European Community and its Member States", 1992. Reprinted in the European Journal of International Law 3 (I), 74-76.

Arel, Dominique. "La face cachée de la révolution orange: l'Ukraine en négation face à son problème régional ", Revue d'études comparatives Est-Ouest 37 (3), 2006, I-38.

Beran, Harry. "A Liberal Theory of Secession ", Political Studies 32, I980, 2 I-3 I.

Brubaker, Rogers. Nationalism Reframed, Cambridge, Cambridge University Press, I996.

- Ethnicity Without Groups, Cambridge, Mass., Harvard University Press, 2004. Buchanan, Allen. "Theories of Secession », Philosophy and Public Affairs, 26 (I), I997, 3I-6I.

—. "Democracy and Secession», in National Self-Determination and Secession, Margaret Moore (ed.), Oxford, Oxford University Press, I998, I4-30.

- Justice, Legitimacy and Self-Determination: Moral Foundations for International Law, Oxford, Oxford University Press, 2003 a.

—. "The Making and Unmaking of Boundaries: What Liberalism Has to Say ", in State, Nations and Borders: The Ethics of Making Boundaries, Allen Buchanan and Margaret Moore (eds), Cambridge, Cambridge University Press, 2003 b, 23I-26I.

Burg, Steven L. and Paul S. Shoup. The War in Bosnia-Herzegovina: Ethnic Conflict and International Intervention, Armonk, NY, M. E. Sharpe, I999.

Campbell, David. National Deconstruction: Violence, Identity and Justice in Bosnia, Minneapolis, University of Minnesota Press, 1998.

Caplan, Richard. Europe and the Recognition of New States in Yugoslavia, Cambridge, Cambridge University Press, 2005.

Catić, Maja. «Moral Claims and Political Demands: The Politics of Genocide Recognition in Bosnia and Ukraine ", Paper presented at the $4^{\text {th }}$ Annual Danyliw Research Seminar in Ukrainian Studies, University of Ottawa, October 23-25, 2008.

Cismas, Ioana. "Secession in Theory and Practice: The Case of Kosovo and Beyond ", Gottingen Journal of International Law 2 (2), 20I0, p. 53 I-587.

Cour internationale de justice. «Conformité au droit international de la déclaration unilatérale d'indépendance relative au Kosovo », La Haye, 22 juillet 20 Io. 
Envoyé spécial du Secrétaire général. Rapport de l'Envoyé spécial du Secrétaire général sur le statut futur du Kosovo. Conseil de sécurité des Nations Unies. (S/2007/I68), 26 mars 2007.

European Community. I99 Ia. "Treaty Provisions for the Convention of November $4^{\text {th }}$ I99I ", Reprinted in The International Conference on the Former Yugoslavia: Official Papers. vol. I, B.G. Racharan (ed.). The Hague: Kluwer Law International, I997.

European Community. I99Ib. "Declaration on Yugoslavia". Extraordinary European Peace Conference Ministerial Meeting, Brussels, I6 December I99I. Reprinted in the European Journal of International Law 3 (I), 73.

European Community. I99Ic. "Guidelines on the Recognition of New States in Eastern Europe and the Soviet Union». Reprinted in the European Journal of International Law 3 (I), 72.

Gagnon, V. P. The Myth of Ethnic Wars: Serbia and Croatia in the 1990s. Ithaca, NY: Cornell University Press, 2004.

Janjić, Dusan, Anna Lalaj and Besnik Pula. "Kosovo under the Milošević Regime». In Confronting the Yugoslav Controversies: A Scholars' Initiative, Charles Ingrao and Thomas A. Emmert (eds.), Purdue: Purdue University Press, 2009, 272-30I.

Judah, Tim. Kosovo: War and Revenge, New Haven, Conn.: Yale University Press, 2002.

Lemarchand, René. "Disconnecting the Threads: Rwanda and the Holocaust Reconsidered ", Journal of Genocide Research 4 (4), 2002, 499-5 I8.

Ministère des Affaires étrangères et européennes. «La France et le Kosovo: Proclamation d'indépendance du Kosovo (I7 fév. 2008)". Paris, République française, I 8 février 2008. [http://www.diplomatie.gouv.fr/fr/pays-zones-geo_833/ kosovo_65o/france-kosovo_460I/proclamation-independance-dukosovor 7.02.08_59650.html\#sommaire_2], consulté le I 5 janvier 2010.

Power, Samantha. A Problem from Hell: America and the Age of Genocide, New York, Basic Books, 2002.

Reyntjens, Filip. "Post-I994 Politics in Rwanda: Problematizing "liberation" and “democratization" ", Third World Quarterly 27 (6), 2006, I I03-I I I 7.

Sémelin, Jacques. Purifier et détruire. Usages politiques des massacres et génocides. Paris, Éditions du Seuil, 2005.

Sobel, Richard. "Portraying American Public Opinion Toward the Bosnia Crisis ", Press and Politics 3 (2), I998, I6-33.

United States Department of State. 2008. «U.S. Recognizes Kosovo as Independent State », 2008/I I 7, Washington DC, State Department [http://200I-2009.state. gov/secretary/rm/2008/02/I00973.htm]. Accessed January I 5, 20 IO.

Valentino, Benjamin. Final Solutions: Mass Killings and Genocide in the 20th Century. Ithaca: Cornell University Press, 2004.

Weinstock, Daniel. «Constitutionalizing the Right to Secede », The Journal of Political Philosophy 9 (2), 2001, I 82-203. 